\title{
Gli1 expression in pancreatic ductal adenocarcinoma and its clinical significance
}

\author{
Y. Abula', C. Yi', X.-Y. Wang', M. Wang', R.-Y. Qin², Y.-Q. Guo', H. Lin ${ }^{1}$ and H.-J. Li ${ }^{3}$ \\ 1'Department of Hepatobiliary Surgery, \\ Affiliated Tumor Hospital of Xinjiang Medical University, Urumqi, China \\ 2Department of Hepatobiliary Surgery, Tongji Hospital, Tongji Medical College, \\ Huazhong University of Science and Technology, Wuhan, Hubei, China \\ ${ }^{3}$ Second Department of General Surgery, Shenzhen Luohu District People's Hospital, \\ Shenzhen, China \\ Corresponding author: H.-J. Li \\ E-mail: Ihjunls@163.com
}

Genet. Mol. Res. 14 (4): 12323-12329 (2015)

Received May 6, 2015

Accepted August 7, 2015

Published October 9, 2015

DOI http://dx.doi.org/10.4238/2015.October.9.21

\begin{abstract}
The aim of this study was to explore the correlation between the expression levels of Gli1 and p53 in pancreatic ductal adenocarcinoma (PDAC) and its pathological significance. Immunohistochemistry $(\mathrm{IHC})$ was employed to measure the expression level of Gli1 and p53 in 85 sets of paraffin-embedded PDAC and corresponding para-carcinoma tissue specimens. The relationship between these results and the respective patients' clinicopathologic parameters was analyzed. IHC staining revealed that the expression levels of Gli1 and p53 in cancer tissues were evidently higher than that of para-carcinoma tissues $(P<0.05)$; while Gli1 expression levels correlated with the corresponding TNM stage and tumor infiltration depth, p53 expression level correlated with the respective TNM stage ( $P$ $<0.05$ ). Taken together, this study demonstrates increased expression
\end{abstract}


of Gli1 and p53 in PDAC, and proves that Gli1 could be apotential biomarker for prognostic judgment.

Key words: Carcinoma; Pancreatic duct; Transcription factor; Prognosis; Factor analysis; Statistics

\section{INTRODUCTION}

Pancreatic ductal adenocarcinoma (PDAC) is a malignancy known for its extremely poor prognosis and a five-year survival rate always less than 5\% (Vasseur et al., 2010; Wang et al., 2014). Its poor prognosis is mainly associated with delayed diagnosis, high degree of malignancy and rapid metastasis. Thus, identification of biomarkers, which can contribute to early diagnosis and prognostic judgment of PDAC is of utmost importance (Kozak et al., 2015; Urayama, 2015). Abnormal activation of Hedgehog $(\mathrm{Hh})$ signal pathway is closely related to the initiation and progression of PDAC (Lee et al., 2014; Konitsiotis et al., 2014). As downstream transcription factors, Gli1 family (including Gli1, Gli2 and Gli3) of proteins play an important role in the Hh signal pathway, with Gli1 being the most important activator (Xu et al., 2014a; Zheng et al., 2015). Previous studies have shown that Gli1 is expressed in several types of tumors and at different stages (Xu et al., 2014b; Zheng et al., 2015). Tumor suppressor p53 plays an equally important role in the development of PDAC as well. Although the existence of mutual suppression circuits of Gli1 and p53 in the neural precursor and glioma stem cells is known, the correlation between Gli1 and p53 in PDAC has not been reported previously. Therefore, we investigated the relationship between Gli1 and p53 and their clinicopathological significance in PDAC.

\section{MATERIAL AND METHODS}

\section{Clinical samples}

The study was approved by the Tumor Hospital of Xinjiang Medical University, China. Tumor excision specimens and corresponding para-carcinoma tissues (controls) were collected from 85 PDAC patients withcomprehensive clinical data between January 2007 and December 2014. The PDAC tissue specimens were subjected to biopsy for confirmation of disease and classified into four stages, stage I (I A+I B), IIA, IIB, and III, according to the 2010 UICC TNM classification system. The complete follow-up data of all the patients were also obtained.

\section{Main or key reagents}

Rabbit anti-human Gli1 and p53 polyclonal antibodies were bought from Santa Cruz Biotechnology, Inc., USA. TRIzol ${ }^{\circledR}$ reagent, PrimerScript RT reagent kit (DRR037S), and SYBR Green II PCR kit were obtained from TaKaRa Bio (Shiga, Japan). Transfection reagent Oligofectamine 2000 was obtained from Invitrogen (Chicago, IL, USA). Gli1 siRNA and siRNA control were synthesized by Shanghai GenePharma Co. Ltd. (Shanghai, China).

\section{IHC and immunoblotting}

The procedures were carried out as described previously (Rossi et al., 2011). Streptavidin- 
peroxidase (SP) method was used in immunohistochemistry (IHC) and the scoring method of Masunaga et al. (2010) was followed for interpretation of the results. The mean of number of positive cells from five random fields (100 cells countedper field) under 400X magnification was calculated. Scores were assigned as follows: $0-9 \%$, score $0 ; 10-24 \%$, score $1 ; 25-49 \%$, score 2; $50-74 \%$, score $3 ; \geq 75 \%$, score 4 . Staining intensity scores were also assigned: pigment-free, score 0 ; pale yellow, score 1; yellow or deep yellow, score 2; brown or dark brown, score 3. A sum of the two scores greater than 2 meant positive expression.

Western blot was performed using the wet transfer method. Protein concentration in the cancer and para-carcinoma tissues, and the pancreatic carcinoma cell lines was determined by BCA protein assay reagent kit (Bioteck, Beijing, China). Samples were separated on 10\% SDSPAGE and transferred onto PVDF membrane. After blocking with $5 \%$ non-fat milk for $2 \mathrm{~h}$, the membrane was incubated with the primary antibody at $4^{\circ} \mathrm{C}$ overnight followed by secondary antibody (TaKaRa Bio, Shiga, Japan) incubation at room temperature for $1.5 \mathrm{~h}$. Images were obtained by chemiluminescence using an imaging instrument (MF-chemibis 3.2 DNR, Israel). The band intensities were quantified with the Quantity One software. The grey value was calculated according to the area and density of the bands from both western blot as well as SDS-PAGE.

\section{Statistics}

All analyses were performed with SPSS 17.0 software. Paired $t$-test was applied to compare protein and mRNA expression between cancer tissues and para-carcinoma tissues. The correlation across the expression levels of different proteins and the that between protein expression and patients' clinicopathological parameters were analyzed by the chi-square test and Pearson correlation analysis, respectively. Kaplan-Meier analysis and log rank-test were used to estimate the cumulative survival rate and the differences in survival probabilities, respectively. Cox multivariate analysis was adopted to assess the independent prognosis factor. $\mathrm{P}<0.05$ was considered as significant difference.

\section{RESULTS}

\section{Expression of Gli1 and p53 in PDAC and para-carcinoma tissues}

As shown in Table 1, whilst the positive expression rates of Gli1 and p53 in PDAC tissues were 51.8 and $56.5 \%$ respectively, those in the para-carcinoma tissues were 29.4 and $25.9 \%$, respectively. Thus, expression rates in the PDAC tissue were significantly higher than those in the para-carcinoma tissues with $\mathrm{P}<0.001$ in both cases. Gli1 was primarily expressed in the nuclei and cytoplasm, whereas p53 was expressed only in the nuclei (Figure 1).

\begin{tabular}{|c|c|c|c|c|}
\hline Specimen & Gli1 (N, \%) & $P$ value & p53 (N, \%) & $P$ value \\
\hline \multirow[t]{2}{*}{ PDAC } & Positive 44 (51.8) & $<0.001$ & Positive 48 (56.5) & $<0.001$ \\
\hline & Negative 41 (48.2) & & Negative 37 (43.5) & \\
\hline \multirow[t]{2}{*}{ Para-cancer } & Positive 25 (29.4) & & Positive 22 (25.9) & \\
\hline & Negative 60 (70.6) & & Negative 63 (74.1) & \\
\hline
\end{tabular}

$(\mathrm{N}=85)$. 


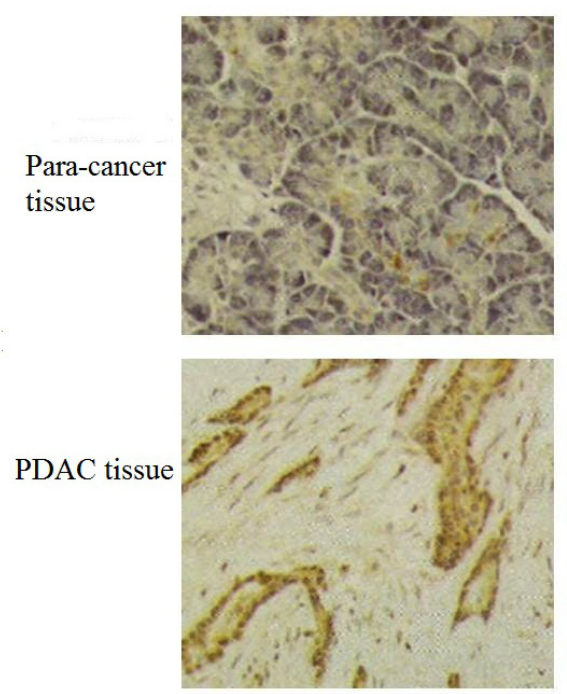

Glil
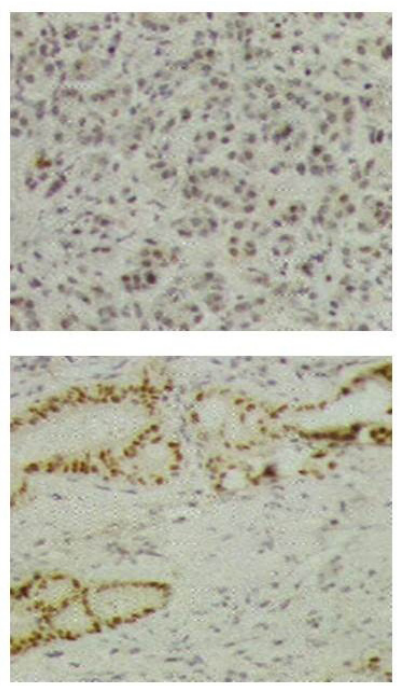

p53

Figure 1. Expression of Gli1 and p53 in PDAC and para-carcinoma tissues. (Immunohistochemistry, 4000X).

\section{Correlation between Gli1 and p53 protein expression and patients' pathological parameters}

As shown in Table 2, the expression level of Gli1 corresponded to the TNM stage and the tumor infiltration depth, and that of p53 corresponded to the TNM stage.

\begin{tabular}{|c|c|c|c|c|c|c|}
\hline \multirow[t]{2}{*}{ Parameters } & \multicolumn{2}{|c|}{ Gli1 } & \multirow[t]{2}{*}{$P$ value } & \multicolumn{2}{|c|}{ p53 } & \multirow[t]{2}{*}{$P$ value } \\
\hline & Negative & Positive & & Negative & Positive & \\
\hline $\mathrm{N}$ & 44 & 41 & & 37 & 48 & \\
\hline Age (Year) & $58.4 \pm 12.2$ & $59.0 \pm 12.5$ & 0.121 & $58.8 \pm 12.4$ & $58.9 \pm 11.3$ & 0.767 \\
\hline Gender (M/F) & $32 / 12$ & $30 / 11$ & 0.293 & $28 / 9$ & $34 / 14$ & 0.314 \\
\hline \multicolumn{7}{|l|}{ Tumor Location } \\
\hline Head of the pancreas & 31 & 29 & 0.191 & 28 & 32 & 0.122 \\
\hline Pancreatic body and tail & 13 & 12 & & 9 & 16 & \\
\hline \multicolumn{7}{|l|}{ Tumor diameter } \\
\hline$<2.5 \mathrm{~mm}$ & 24 & 22 & 0.220 & 21 & 25 & 0.201 \\
\hline$\geq 2.5 \mathrm{~mm}$ & 20 & 19 & & 16 & 13 & \\
\hline \multicolumn{7}{|l|}{ Tumor differentiation } \\
\hline Poor & 12 & 10 & 0.113 & 9 & 13 & 0.143 \\
\hline Moderate & 21 & 22 & & 18 & 25 & \\
\hline High & 11 & 9 & & 10 & 10 & \\
\hline \multicolumn{7}{|l|}{ Tumor Invasion } \\
\hline $\mathrm{T} 1+\mathrm{T} 2$ & 21 & 10 & 0.013 & 18 & 13 & 0.121 \\
\hline $\mathrm{T} 3+\mathrm{T} 4$ & 23 & 31 & & 19 & 35 & \\
\hline \multicolumn{7}{|l|}{ Lymph node metastasis } \\
\hline No & 34 & 32 & 0.442 & 31 & 35 & 0.153 \\
\hline Yes & 10 & 9 & & 6 & 13 & \\
\hline \multicolumn{7}{|l|}{ TNM stage } \\
\hline$I+\| A$ & 35 & 28 & 0.022 & 32 & 31 & 0.015 \\
\hline$\|\mathrm{II}+\| \mathrm{I}$ & 9 & 13 & & 5 & 17 & \\
\hline
\end{tabular}




\section{Correlation between expression levels of Gli1 and p53}

According to Pearson correlation analysis, the expression levels of Gli1 and p53 did not correlate with each other $(r=0.104, P=0.124)$.

\section{Relationship between PDAC prognosis and the expression levels of Gli1 and p53}

According to Cox regression analysis, after adjustment of other confounders, the expression level of Gli1 was found to be an independent factor for the prognosis of PDAC (RR = $2.432,95 \% \mathrm{Cl}=1.191-4.881, \mathrm{P}=0.002)($ Table 3$)$.

Table 3. Cox regression results of protein expression levels and PDAC prognosis.

\begin{tabular}{lccccrr}
\hline Risk factors & $\beta$ value & SE & Wald value & P value & RR & $95 \% \mathrm{Cl}$ \\
\hline Gli1 expression & 0.801 & 0.379 & 4.123 & 0.002 & 2.432 & $1.191-4.881$ \\
Tumor invasion & 0.657 & 0.388 & 3.976 & 0.013 & 2.012 & $1.091-4.120$ \\
TNM stage & 0.701 & 0.354 & 4.014 & 0.009 & 2.011 & $1.103-4.776$ \\
\hline
\end{tabular}

\section{DISCUSSION}

The abnormal activation of Hh signal pathway play a key role in the development and progression of PDAC. In the initial stage of PDAC, pancreatic ductal epithelial cells develop pancreatic intraepithelial neoplasia (PanIN), which is the precancerous lesion of invasive pancreatic cancer. Analysis of specimens from human pancreatic carcinoma revealed that during the transition from PanINto invasive pancreatic carcinoma, the expression level of members of Hh pathway such as $\mathrm{SHH}$ and Ptcl were increased. Transgenic mice with constitutively expressed $\mathrm{SHH}$ under specific promoter $\mathrm{Pdx}-1$ indicated that $\mathrm{SHH}$ was abnormally expressed in the pancreatic endothelium of transgenic mice. In this case, hyperactivated Hh pathway played an important role in the early stage of PDAC implicating that control of Hh pathway activation was important to prevent abnormal proliferation and tumorigenesis.

Berman et al. (2003) found that Hh pathway was abnormally activated in various gastrointestinal tumors including gastric carcinoma, esophageal cancer, cholangiocarcinoma, and pancreatic carcinoma (except colon cancer). On blocking the activity of $\mathrm{Hh}$ ligand on cell membrane by an anti-Hh antibody in pancreatic carcinoma cell lines, the growth was restrained. These evidences implicate that the stimulation of Hh ligand was necessary for the proliferation of pancreatic carcinoma cells.

Previous studies have also shown that Gli1 mRNA expression was higher in the pancreatic carcinoma tissues than para-carcinoma tissues (Guo et al., 2007; Satoh et al., 2008). These reports suggested that the expression level of Gli1 mRNA was associated with tumor differentiation and lymphatic metastasis. However, this study provides evidence that Gli1 and p53 are highly expressed in PDAC and that the expression level of Gli1 correlates with pancreatic carcinoma infiltration depth as well as TNM stage. This was also consistent with the expression of Gli1 in head and neck squamous cell carcinoma and liver cancer. The disparity with works by Guo et al. (2007) and Satoh et al. (2008) could be due to the differences in research methods and sample size. 
Abe et al. (2008) reported that the increase of Gli1 in C3H10T1/2 cells inhibited the activity of $p 53$ by activating MDM2, a negative regulator of p53. Stecca and Ruiz i Altaba (2009) also found that the activity of MDM2 was strongly enhanced in the brain of Gli1 transgenic mice. Furthermore, in the cerebellar granule neuron precursors of MDM2 knockout mice, Hh signal was accompanied by the reduction of Gli1 and Gli12 expression. Thus, a positive regulation between Gli1 and MDM2 is evident in normal as well as tumor tissues in several organs.

Our study showcases that PDAC patients with positive expression of Gli1 had poorer prognosis compared to those with negative expression, which was an independent risk factor for the prognosis of PDAC. Our results are similar to those from glioma, mammary cancer and SCCHN patients who displayed high expression level of Gli1 and poor prognosis (Zhuang et al., 2013; Xin et al., 2014).

In summary, our results highlight that Gli1 facilitates the invasion and metastasis of PDAC cells indicated by its correlation with PDAC infiltration depth and TNM stage. Since this may be one of the primary causes which lead to the poor prognosis of PDAC, Gli1 could be probably be used as a biomarker to improve prognosis.

\title{
Conflicts of interest
}

The authors declare no conflict of interest.

\section{ACKNOWLEDGMENTS}

\author{
Research supported by the Xinjiang Uygur Autonomous Region Science and Technology \\ Support Project (\#201291170).
}

\section{REFERENCES}

Abe $\mathrm{Y}$, Oda-Sato $\mathrm{E}$, Tobiume K, Kawauchi K, et al. (2008). Hedgehog signaling overrides p53-mediated tumor suppression by activating Mdm2. Proc. Natl. Acad. Sci. U. S. A. 105: 4838-4843.

Berman DM, Karhadkar SS, Maitra A, Montes De Oca R, et al. (2003). Widespread requirement for Hedgehog ligand stimulation in growth of digestive tract tumours. Nature 425: 846-851.

Guo JF, Li ZS, Jin ZD, Gao J, et al. (2007). mRNA expression of GLI1 in pancreatic carcinoma and clinical significance thereof. Zhonghua Yi Xue Za Zhi 87: 826-828.

Konitsiotis AD, Chang SC, Jovanović B, Ciepla P, et al. (2014). Attenuation of hedgehog acyltransferase-catalyzed sonic Hedgehog palmitoylation causes reduced signaling, proliferation and invasiveness of human carcinoma cells. PLoS One 9: e89899.

Kozak G, Blanco FF and Brody JR (2015). Novel targets in pancreatic cancer research. Semin. Oncol. 42: 177-187.

Lee JJ, Perera RM, Wang H, Wu DC, et al. (2014). Stromal response to Hedgehog signaling restrains pancreatic cancer progression. Proc. Natl. Acad. Sci. U. S. A. 111: E3091-E3100.

Masunaga R, Kohno H, Dhar DK, Ohno S, et al. (2000). Cyclooxygenase-2 expression correlates with tumor neovascularization and prognosis in human colorectal carcinoma patients. Clin. Cancer Res. 6: 4064-4068.

Rossi M, Magnoni L, Miracco C, Mori E, et al. (2011). $\beta$-catenin and Gli1 are prognostic markers in glioblastoma. Cancer Biol. Ther. 11: 753-761.

Satoh K, Kanno A, Hamada S, Hirota M, et al. (2008). Expression of Sonic hedgehog signaling pathway correlates with the tumorigenesis of intraductal papillary mucinous neoplasm of the pancreas. Oncol. Rep. 19: 1185-1190.

Stecca B and Ruiz i Altaba A (2009). A GLI1-p53 inhibitory loop controls neural stem cell and tumour cell numbers. EMBO J. 28: 663-676.

Urayama S (2015). Pancreatic cancer early detection: expanding higher-risk group with clinical and metabolomics parameters. World J. Gastroenterol. 21: 1707-1717.

Vasseur S, Tomasini R, Tournaire R and lovanna JL (2010). Hypoxia induced tumor metabolic switch contributes to pancreatic cancer aggressiveness. Cancers 2: 2138-2152. 
Wang H, Estrella JS, Chatterjee D, Katz MH, et al. (2014). Hepatobiliary/Pancreas Pathology: SY11-3 pancreatic ductal adenocarcinoma: neoadjuvant therapies and pathology. Pathology 46 (Suppl 2): S25.

Xin Y, Shen XD, Cheng L, Hong DF, et al. (2014). Perifosine inhibits S6K1-Gli1 signaling and enhances gemcitabine-induced anti-pancreatic cancer efficiency. Cancer Chemother. Pharmacol. 73: 711-719.

Xu X, Su B, Xie C, Wei S, et al. (2014a). Sonic hedgehog-Gli1 signaling pathway regulates the epithelial mesenchymal transition (EMT) by mediating a new target gene, S100A4, in pancreatic cancer cells. PLoS One 9: e96441.

Xu Y, An Y, Wang X, Zha W, et al. (2014b). Inhibition of the Hedgehog pathway induces autophagy in pancreatic ductal adenocarcinoma cells. Oncol. Rep. 31: 707-712.

Zheng X, Song T, Dou C, Jia Y, et al. (2015). CtBP2 is an independent prognostic marker that promotes GLI1 induced epithelial-mesenchymal transition in hepatocellular carcinoma. Oncotarget 6: 3752-3769.

Zhuang Z, Wang K, Cheng X, Qu X, et al. (2013). LKB1 inhibits breast cancer partially through repressing the Hedgehog signaling pathway. PLoS One 8: e67431. 